\title{
Channeling implantation of high energy carbon ions in a diamond crystal: Determination of the induced crystal amorphization
}

\author{
M. Ericha,*, M. Kokkoris ${ }^{b}$, S. Fazinićc ${ }^{a}$ and S. Petrovića \\ aLaboratory of Physics, "Vinča" Institute of Nuclear Science, University of Belgrade, PO Box 552, Belgrade, Serbia \\ ${ }^{b}$ Department of Physics, National Technical University of Athens, Zografou Campus 157 80, Athens, Greece \\ cLaboratory for Ion Beam Interactions, Department of Experimental Physics, Institute Ruđer Bošković, Bijenička \\ cesta 54, 10000 Zagreb, Croatia
}

\begin{abstract}
This work reports on the induced diamond crystal amorphization by $4 \mathrm{MeV}$ carbon ions implanted in the $<100>$ oriented crystal and its determination by application of RBS/C and EBS/C techniques. The spectra from the implanted samples were recorded for $1.2,1.5,1.75$ and $1.9 \mathrm{MeV}$ protons. For the two latter ones the strong resonance of the nuclear elastic scattering ${ }^{12} \mathrm{C}\left(\mathrm{p}, \mathrm{p}_{0}\right){ }^{12} \mathrm{C}$ at $1.737 \mathrm{MeV}$ was explored. The backscattering channeling spectra were successfully fitted and the ion beam induced crystal amorphization depth profile was determined using a phenomenological approach, which is based on the properly defined Gompertz type dechanneling functions for protons in the $<100>$ diamond crystal channels and the introduction of the concept of ion beam amorphization, which is implemented through our newly developed computer code CSIM.
\end{abstract}

*Corresponding author. Tel: +381 11644 7700. Email: marko.erich@gmail.com 


\section{Introduction}

Diamond is a semiconductor whose physical properties can be important for technological applications, among which the production of detectors is the most promising one. Namely, in comparison with silicon, diamond has a higher band gap (5.45 eV), while exhibiting lower dark current and breakout field $\left(>1 \mathrm{kV}^{-1}\right)$ [1], higher resistance to damage due to its strong chemical bonds and very high thermal conductivity, $22 \mathrm{Wcm}^{-1} \mathrm{~K}^{-1}$, for natural, and, $33 \mathrm{Wcm}^{-1} \mathrm{~K}^{-1}$, for synthetic diamond, respectively [2]. For high-purity diamond crystals, grown using the chemical vapor deposition technique (CVD), the electron and hole room temperature drift mobility was reported to be as high as $4500 \mathrm{~cm}^{2} \mathrm{~V}^{-1} \mathrm{~s}^{-1}$ and $3800 \mathrm{~cm}^{2} \mathrm{~V}^{-1} \mathrm{~s}^{-1}$ [3] respectively. Additionally, CVD grown diamond detectors show enhanced performance characteristics, such as, extraordinary long lifetimes and very high mobility of charge carriers [4, 5]. Based on these properties, diamond detectors can be used for the detection of light charged particles $[1,6,7]$, fission fragments [8], neutrons [9], ultra-violet [10] and X-ray [11] emission. Recently, diamond detectors have shown great promise for the production of photonic devices [12]. Also, they constitute an excellent choice for radiotherapy dosimetry owing to their soft tissue equivalence $(Z=6)$ [13].

It is well known that the ion implantation is a standard method for the introduction of dopants in semiconductors [14]. One of the drawbacks of the process is the induced amorphization of the crystal $[15,16]$. However, the induced amorphization can even be favorable if the so called pre-amorphization process is required. In the case of diamond based devices amorphized diamond layers are observed to have increased electric conductivity, making it suitable for ohmic contacts $[17,18]$. In broader sense, preamorphization of a semiconductor substrate in the contemporary semiconductor industry allows for production of the ultra-shallow junctions for advanced complementary metal oxide semiconductor transistors (CMOS), which leads to significantly lower parasitic capacities and, consequently, faster integrated circuits [19]. In this aspect, a good quantification technique for determination of the amorphization depth profile in semiconductors is emerging as an imperative. It should be noted here that the techniques for characterization of the ion beam induced amorphization, reported in the past, like Scanning Transmission Electron Microscopy (STEM), which require extensive sample 
preparations and rigid experiment conditions [20], or micro Raman spectroscopy, which needs sample cut along longitudinal axis in order to assess amorphization depth profile [21], are dominantly qualitatively ones with respect to a precise determination of the noncrystalline phase induced by the ion beam implantation. Further, Positron Annihilation Spectroscopy (PAS) gives point defects depth profile with a suitable probing positron beam [22] while X-ray diffraction (XRD) is a nondestructive method that gives averages over depth, though a lot has been done to increase XRD resolution [23]. It is also well known that the Ion Beam Analysis (IBA) techniques: Rutherford Backscattering Spectroscopy (RBS), Elastic Backscattering Spectroscopy (EBS), Elastic Recoil Detection Analysis (ERDA) and Nuclear Reaction Analysis (NRA), can determine the depth profile of impurities in a bulk material with a depth resolution in the range of a few nanometers [24]. Subset of those techniques in the channeling crystal orientation, RBS/C and EBS/C, with lowered backscattered signal originating from the bulk crystal can additionally provide the ion beam induced amorphization depth profiling information on the bulk crystalline material $[25,26]$. However, the accurate quantification of the obtained amorphization profiles [27] still constitutes an open problem. In the present work our main goal is to obtain quantified amorphization depth profile induced by $\mathrm{MeV}$ ion implantation by means of fitting experimental RBS/C and EBS/C spectra using in-house developed computer simulation code which takes into account the background yield of bulk crystal material in the RBS/C or EBS/C spectra [28].

\section{Computer simulation code}

A computer simulation code for the description of RBS and EBS spectra in the random and channeling mode, called CSIM, was developed recently [28]. It is based on a phenomenological approach [28]. In the channeling mode, the code uses a Gompertz type sigmoidal dechanneling function, with the fitting parameters, $\mathrm{k}$ and $\mathrm{x}_{\mathrm{c}}$, being the dechanneling rate and range, respectively. The additional fitting parameter $\alpha$ is the ratio of the stopping powers of the channeled to random ions. The target in the code is divided into computational layers, whose thickness can be adjusted. Properties of the target layer i.e. the atom types, stoichiometry and density, can be set as an input in the code. 
The ion beam in the layer is divided in channeled and dechanneled parts. The dechanneled part is treated as being random and consequently its ion beam energy loss/stopping power is calculated by Ziegler et al. stopping data [29]. For the channeled part of the ion beam, it has been assumed that the energy loss/stopping power is less than the random one by a factor determined by the parameter $\alpha$. The same holds for the energy loss straggling, which is calculated as a sum of a part dependent on the energy loss and a statistical one [28]. The statistical part is calculated by using Yang's formula [30]. The backscattering cross section is obtained from the IBANDL database for a certain backscattering angle [31]. It takes into account the Rutherford and non-Rutherford elastic scattering processes including the existing nuclear resonances.

In a target layer, the dechanneled/random backscattered part of the ion beam along a certain angle is calculated from the assumed phenomenological dechanneling function sigmoidal Gompertz function, and the backscattering elastic differential cross section. Then it is followed back through the target towards the detector having the total (calculated) energy loss and straggling at the exit of the target. The detector resolution is taken into account assuming that the energy loss of the detected ion is of a Gaussian form, whose standard deviation is the result of the sum in quadrature of standard deviations corresponding to the energy loss straggling and to the resolution of the detector. The remaining channeled and dechanneled parts of the beam proceed to the next layer, in which the new, modified channeled and dechanneled parts are calculated and the whole procedure is repeated. It should be mentioned here that one can manually introduce the fitting channeling parameters $-\mathrm{k}, \mathrm{x}_{\mathrm{c}}$ and $\alpha$ in each target layer. However, in this work, it has been assumed that the fitting channeling parameters are the same in every target layer and are determined via a $\chi^{2}$ minimization using the MINUIT minimization routine developed at CERN [32].

\section{Results}

In this work $4 \mathrm{MeV}$ carbon ions were implanted in a diamond crystal in the $<100>$ axial channeling direction, with a fluence of $\sim 10^{16} \mathrm{ions} / \mathrm{cm}^{2}$. RBS/C and EBS/C spectra were taken on the implanted spots and on a virgin spot of the diamond crystal by using 1.2, 1.5, 
and, 1.75 and $1.9 \mathrm{MeV}$ protons. In the EBS/C spectra the strong resonance of the nuclear elastic scattering ${ }^{12} \mathrm{C}\left(\mathrm{p}, \mathrm{p}_{0}\right){ }^{12} \mathrm{C}$ at $\mathrm{E}_{\mathrm{p}, \mathrm{lab}}=1.737 \mathrm{MeV}$ was explored [31].

The experiments were performed at Ruđer Bošković Institute, Zagreb, Croatia. The ion beams were produced from the $1 \mathrm{MV}$ HV Tandetron accelerator. The goniometer was installed on the beam line allowing for a 5-degree freedom of movement with an angular precision of $0.01^{0}$. The beam line had a collimating system composed of two small apertures separated by $25 \mathrm{~cm}$. The silicon detector (SSB) was set at $160^{\circ}$. The beam spot had a size of $0.8 \mathrm{~mm}^{2}$. All the artificial diamonds were flat, polished and rectangular $3 \times 3$ $\mathrm{mm}^{2}$ Element 6 synthetic, high-purity single crystals (with boron and nitrogen dopant concentrations of less than $0.05 \mathrm{ppm}$ and $1 \mathrm{ppm}$ respectively), cut in the [100] direction. The diamonds were $0.3 \mathrm{~mm}$ thick, with flat edges (tolerance $<0.2 \mathrm{~mm}$ ), grown by Chemical Vapor Deposition (CVD) synthesis processes. The diamond crystal alignment along the $<100>$ crystal axis was performed with $1 \mathrm{MeV}$ protons [28]. In order to minimize the crystal lattice damage, and avoid any type of heating effects, the beam current on target never exceeded $\sim 4 \mathrm{nA}$.

In order to explain the experimental results and determine the ion beam induced crystal amorphization the CSIM computer code [28] was modified by introducing an additional fitting parameter $\mathrm{A}$, the ratio of the amorphous to the crystalline phase. Then, in the calculation, for a particular value of variable $A$ in the layer, the part of the beam corresponding to the value of $\mathrm{A}$ is treated as being dechanneled/random, thus decreasing the percentage of the channeled part of the beam in the layer. In the first approximation, which is used in this work, it will be assumed that the dechanneling and the induced amorphization processes are independent. This assumption can be justified by the fact that implanted doses cause relatively small crystal damage in the trace region as has been previously observed in the studies performed with 6H-SiC crystal [33]. This means that one can use the dechanneling parameters obtained for the virgin crystal and independently introduce the induced amorphization depth profile. This then allows for relative easy spectrum fitting by changing number, height and width of bars comprising amorphization depth profile until suitable fit is obtained. Having all three parameters free to change in each part of amorphization profile creates too many parameters for minimization to make 
RBS/C or EBS/C technique practical for amorphization depth profiling. However, using channeling parameters that do not differ too much from ones obtained in virgin crystal case is an option which we would like to offer to potential future users. True testing of channeling parameter dependence on amount of amorphization in the crystal would require obtaining exact quantified amorphization depth profile via some other method and then using that profile to assess how much channeling parameters differ with amount of amorphization phase in each layer, which is out of the scope of this paper. It should be also noted that the dependence of the energy loss process on the induced amorphization is implicit. Namely, the obtained increase of the random part of the beam induced by the amorphization accordingly increases the ion energy loss. The ion energy loss straggling has also been changed with the induced amorphization in accordance to the change of the ion energy loss.

Therefore, in this work, the fitting procedure of the experimental spectrum assumes the introduction of the already obtained dechanneling parameters $\mathrm{k}$ and $\mathrm{x}_{\mathrm{c}}$, and $\alpha$, for the virgin $<100>$ diamond crystal case [28]. For the proton energies under consideration these parameters are given in Table 1, which demonstrates the right trend of the parameters $\mathrm{k}$ and $x_{c}$ with energy ( $k$ is decreasing and $x_{c}$ is increasing), whereas, the parameter $\alpha$ can be considered as constant for all practical purposes. Furthermore, in the fitting procedure, the parameter $\mathrm{A}$ has been adjusted in each crystal layer as to obtain the best fitting curve of the experimental spectrum. As a result the amorphization depth profile has been determined.

Figs. 1(a) and (b) show the RBS/C spectra and the fitting curves for the proton energies of 1.2 and $1.5 \mathrm{MeV}$, whereas, Figs. 1(c) and (d) show the EBS/C spectra and the fitting curves for the proton energies of 1.75 and $1.9 \mathrm{MeV}$, for both the virgin and channeling implantation $<100>$ diamond crystal cases. The experimental data are designated by black and open dots and the fitting curves by blue and red lines, for the implanted and virgin diamond crystal cases, respectively. The induced amorphization is clearly visible as the difference of the implanted and virgin parts of the spectra. Also, EBS/C spectra clearly show the resonance at $E_{p, l a b}=1.737 \mathrm{MeV}$, in the trace region for $1.75 \mathrm{MeV}$ protons, and inside the crystal for $1.9 \mathrm{MeV}$ protons. 
The corresponding amorphization depth profiles are shown in Figs. 2(a)-(d), respectively. It is clear that fits of the experimental data presented in Fig. 1 are excellent. Also, the obtained amorphization depth profiles, presented in Fig. 2, are consistent with respect to the fact that they all show a practically non-disturbed trace region of about 1.5 $\mu \mathrm{m}$ in thickness and a highly non-symmetric peak structure with the position of the peak being located at the same position, which is in all the cases equal to $2.1 \mu \mathrm{m}$, accompanied by a tail reaching up to $\sim 5 \mu \mathrm{m}$ in depth, as expected due to the channeling implantation. It should be noted here that the amorphization layer at the surface was introduced in order to simulate the crystal lattice's blocking pattern from the planes forming the $<100>$ channel, which leads to increased proton backscattering in the channeling direction from the crystal surface contributing to the surface channeling peak. This, in principle, depends on the initial ion beam energy. Also, the observed differences between the amorphization depth profiles for different energies, particularly the ones for the proton energies of 1.2 and $1.9 \mathrm{MeV}$, when the values of the maximum induced amorphizations are equal to $70 \%$ and $50 \%$, respectively, can be attributed to the uncertainties of the stopping power, energy loss straggling, dechanneling parameters and non-Rutherford/resonant elastic differential cross sections.

In the final analysis, the amorphization depth profiles for all proton energies were averaged. This step was chosen to minimize the influence of the uncertainties of all the processes involved, as already mentioned above, and to demonstrate the consistency of the applied phenomenological approach. The result is shown in Fig. 3. The thus obtained undisturbed trace region was equal to $1.55 \mu \mathrm{m}$, the position of the maximum amorphization was equal to $2.1 \mu \mathrm{m}$, with the pronounced tail distribution extending up to 5 $\mu \mathrm{m}$ as mentioned above. Using these averaged values as input in the CSIM code, the reproduction of the experimental channeling spectra is also excellent and does not deviate from the $\chi^{2}$ minimization one by more than $1-5 \%$, which justifies our approach.

\section{Conclusions}

It is shown here that it is possible to determine the carbon ion beam induced amorphization depth profile of the diamond crystal by applying the modified 
phenomenological channeling model for fitting the RBS/C and EBS/C spectra. This could open the usage of RBS/C and EBS/C techniques (non-destructive, non-biohazard, fast and well known) for quantified crystal amorphization (damage) depth profiling. The newly developed CSIM code can be additionally easily adapted for the treatment of implanted impurity ions, something which will be the aim of a future work. The beta version of the code will be offered for downloading and testing by the scientific community in the near future via the Vinča Institute's web site.

As a result, it has been demonstrated that $\mathrm{MeV}$ range implanted carbon ions in the channeling mode can produce a sharp deeply buried carbon amorphization layer in a diamond crystal, with the trace region practically undisturbed. This could be compared with a recent report about the implementation of the plasma-enhanced CVD method for producing freestanding diamond films via the buried graphite interlayer at the interface between the diamond film and the Ni substrate [34].

\section{Acknowledgements}

ME and SP acknowledge the support by the Ministry of Education, Science and Technological Development of Serbia through project "Physics and Chemistry with Ion Beams", No. III45006. ME, SP and MK acknowledge that this work is part of the NTUA//VINS Collaboration Agreement. MK and SP acknowledge partial support by the Horizon 2020 project AIDA-2020, GA No. 654168. SF acknowledges the support of the Croatian Science Foundation under the project MIOBICC (No. 8127). 


\section{References}

[1] F. Schirru, D. Chokheli and M. Kis, "Thin single crystal diamond detectors for alpha particle detection", Diamond \& Related Mat., 49, pp. 96-102 (2014).

[2] T.R. Anthony, W.F. Banholzer, J.F. Fleischer, L. Wei, P.K. Kuo, R.L. Thomas, et al., “Thermal diffusivity of isotopically enriched ${ }^{12}$ C diamond", Phys. Rev. B, 42, pp. 1104-1111 (1990).

[3] J. Isberg, J. Hammersberg, E. Johansson, T. Wilkstoem, D.J. Twichen, A.J. Whitehead, et al., "High Carrier Mobility in Single-Crystal Plasma-Deposited Diamond", Science, 297, pp. 1670-1672 (2002).

[4] M. Pomorski, B. Caylar, and P. Bergonzo, "Super-thin single crystal diamond membrane radiation detectors", Appl. Phys. Lett., 103, 112106 (2013).

[5] S. Almaviva, M. Marinelli, E. Milani, G. Prestopino, A. Tucciarone, C. Verona, et al., "Chemical vapor deposition diamond based multilayered radiation detector: Physical analysis of detection properties“ J. Appl. Phys., 107, 014511 (2010).

[6] E. Berdermann, M. Pomorski, W. de Boer, M. Ciobanu, S. Dunst, C. Grah, M. Kis, W. Koenig, W. Lange, et al., “Diamond detectors for hadron physics research”, Diamond \& Related Mat., 19, pp. 358-367 (2010).

[7] S. Wang, P. Sellin and A. Lohstroh, "Alpha particle transient response of a polycrystalline diamond detector", Carbon, 43, pp. 3167-3171 (2005).

[8] S. Oberstedt, R. Borcea, T. Brys, T. Gamboni, W. Geerts, F.J. Hambsch, et al., “Artificial diamonds as radiation-hard detectors for ultra-fast fission-fragment timing" Nucl. Instr. Meth. A, 714, pp. 31-37 (2013).

[9] A. Kumar, A. Singh, A. Kumar and A. Topkar, "Fabrication and characterization of polycrystalline diamond detectors for fast neutron monitoring“, Nucl. Instr. Meth. A, 785, pp. 55-60 (2015). 
[10] M. Liao, X. Wang, T. Teraji, S. Koizumi and Y. Koide, "Light intensity dependence of photocurrent gain in single-crystal diamond detectors", Phys. Rev. B, 81, 033304 (2010).

[11] J. Morse, M. Salome, E. Berdermann, M. Pomorski, W. Cunningham and J. Grant, "Single crystal CVD diamond as an X-ray beam monitor", Diamond \& Related Mat., 16, pp. 10491052 (2007).

[12] I. Aharonovich, A.D. Greentree and S. Prawer, "Diamond photonics", Nature Photonics, 5, pp. 397 (2011).

[13] J.M. Larraga-Gutierrez, P. Ballesteros-Zebadua, M. Rodriguez-Ponce, O.A. GarciaGarduno, and 0.0. Galvan de la Cruz, "Properties of a commercial PTW-60019 synthetic diamond detector for the dosimetry of small radiotherapy beams“, Phys. Med. Biol., 60, pp. 905-924 (2015).

[14] M. Nastasi, J. W. Mayer, Ion Implantation and Synthesis of Materials, Springer-Verlag, New York, 2006.

[15] J.D. Hunn, S.P. Withrow, C.W. White and D.M. Hembree, "Raman scattering from MeVion implanted diamond", Phys. Rev. B, 52, pp. 8106-8111 (1995).

[16] B.A. Fairchild, S. Rubanov, D.W.M. Lau, M. Robinson, I. Suarez-Martinez, N. Marks, et al., "Mechanism of amorphization in diamond", Adv. Mater., 24, 2024-2029 (2012).

[17] Y.G. Chen, M. Hasegawa, H. Okushi, S. Koizumi, H. Yoshida, T. Sakai and N. Kobayashi, "Electrical properties of graphite/homoepitaxial diamond contacts", Diamond \& Related Mat., 11, pp. 451-457 (2002).

[18] Y. Avigal, V. Richter, B. Fizgeer, C. Saguy and R. Kalish, “The nature of ion-implanted contacts to polycrystalline diamond films", Diamond \& Related Mat., 13, pp. 1674-1679 (2004).

[19] T. Noda, C. Vrancken, and W. Vandervorst, "Modeling of junction formation in scaled Si devices", J. Comput. Electron., 13, pp. 33-39 (2014). 
[20] D.A. Muller and J. Grazul, "Optimizing the environment for sub-0.2 nm scanning transmission electron microscopy", J. Electron Microsc., 50, pp. 219-226 (2001).

[21] M. Erich, S. Petrović, M. Kokkoris, E. Liarokapis, A. Antonakos and I. Telečki, “MicroRaman depth profiling of silicon amorphization induced by high-energy ion channeling implantation“, J. Raman Spectrosc., 44, pp. 496-500 (2013).

[22] R.W. Siegel, "Positron Annihilation Spectroscopy”, Ann. Rev. Mater. Sci., 10, pp. 393-425 (1980)

[23] P. Thibault and V. Elser, "X-ray diffraction microscopy", Ann. Rev. Condens. Mater. Phys., 1, pp. 237-255 (2010).

[24] M. Nastasi, J. Mayer and Y. Wang, Ion Beam Analysis: Fundamentals and Applications, Boca Raton, Taylor \& Francis, CRC Press, 2014.

[25] J. Nakata, "Annealing of ion-implanted defects in diamond by MeV ion-beam irradiation", Phys. Rev. B, 60, pp. 2747-2761 (1999).

[26] D.N. Jamieson, S. Prawer, S.P. Dooley and R. Kalish, "Regrowth zones in laser annealed, radiation damaged diamond", Nucl. Instr. Meth. B, 77, pp. 457-462 (1993).

[27] M. Erić, S. Petrović, M. Kokkoris, A. Lagoyannis, V. Paneta, S. Harrisopulos and I. Telečki, "Depth profiling of high energy nitrogen ions implanted in the $\langle 100\rangle,\langle 110\rangle$ and randomly oriented silicon crystals", Nucl. Instr. Meth. B, 274, pp. 87-92 (2012).

[28] M. Erich, M. Kokkoris, S. Fazinić, and S. Petrović, “EBS/C proton spectra from a virgin diamond crystal", Nucl. Instr. Meth. B, 381, pp. 96-102 (2016).

[29] J. Ziegler, J. Biersack and U. Littmark, "The Stopping and Range of Ions in Solids, vol. 1", Pergamon Press Inc, New York (1985).

[30] Q. Yang, D. O'Connor and Z. Wang, “Empirical formulae for energy loss straggling of ions in matter“, Nucl. Instr. Meth. B, 61, pp. 149-155 (1991). 
[31] A. F. Gurbich, "Ion Beam Analysis Nuclear Data Library" [Online]. Available: https://www-nds.iaea.org/exfor/ibandl.htm.

[32] F. James, M. Roos, "Minuit - a system for function minimization and analysis of the parameter errors and correlations", Comput. Phys. Commun., 10, pp. 343-367 (1975).

[33] X. Chen, W. Zhou, Q. Feng, J. Zheng, X. Liu, et al., "Irradiation effects in 6H-SiC induced by neutron and heavy ions: Raman spectroscopy and high-resolution XRD analysis", J. Nucl. Mater., vol. 478, pp. 215-221 (2016).

[34] S. Ito, M. Nagai, T. Matsumoto, T. Inokuma, and N. Tokuda, "Self-separation of freestanding diamond films using graphite interlayers precipitated from $\mathrm{C}$-dissolved $\mathrm{Ni}$ substrates“, J. Crys. Growth, 470, pp. 104-107 (2017). 


\section{Table 1}

\begin{tabular}{|c|c|c|c|c|}
\hline $\mathrm{E}[\mathrm{MeV}]$ & 1.20 & 1.50 & 1.75 & 1.90 \\
\hline $\mathrm{k}\left[\mu \mathrm{m}^{-1}\right]$ & 0.481 & 0.400 & 0.300 & 0.197 \\
\hline $\mathrm{x}_{\mathrm{c}}[\mu \mathrm{m}]$ & 2.050 & 2.470 & 2.749 & 4.160 \\
\hline$\alpha$ & 0.880 & 0.883 & 0.900 & 0.865 \\
\hline
\end{tabular}

Table 1. Values of the parameters $k, x_{c}$ and $\alpha$, for proton energies of $1.2,1.5,1.75$ and 1.9 $\mathrm{MeV}$, for the virgin $<100>$ diamond crystal. 


\section{Figure 1a}

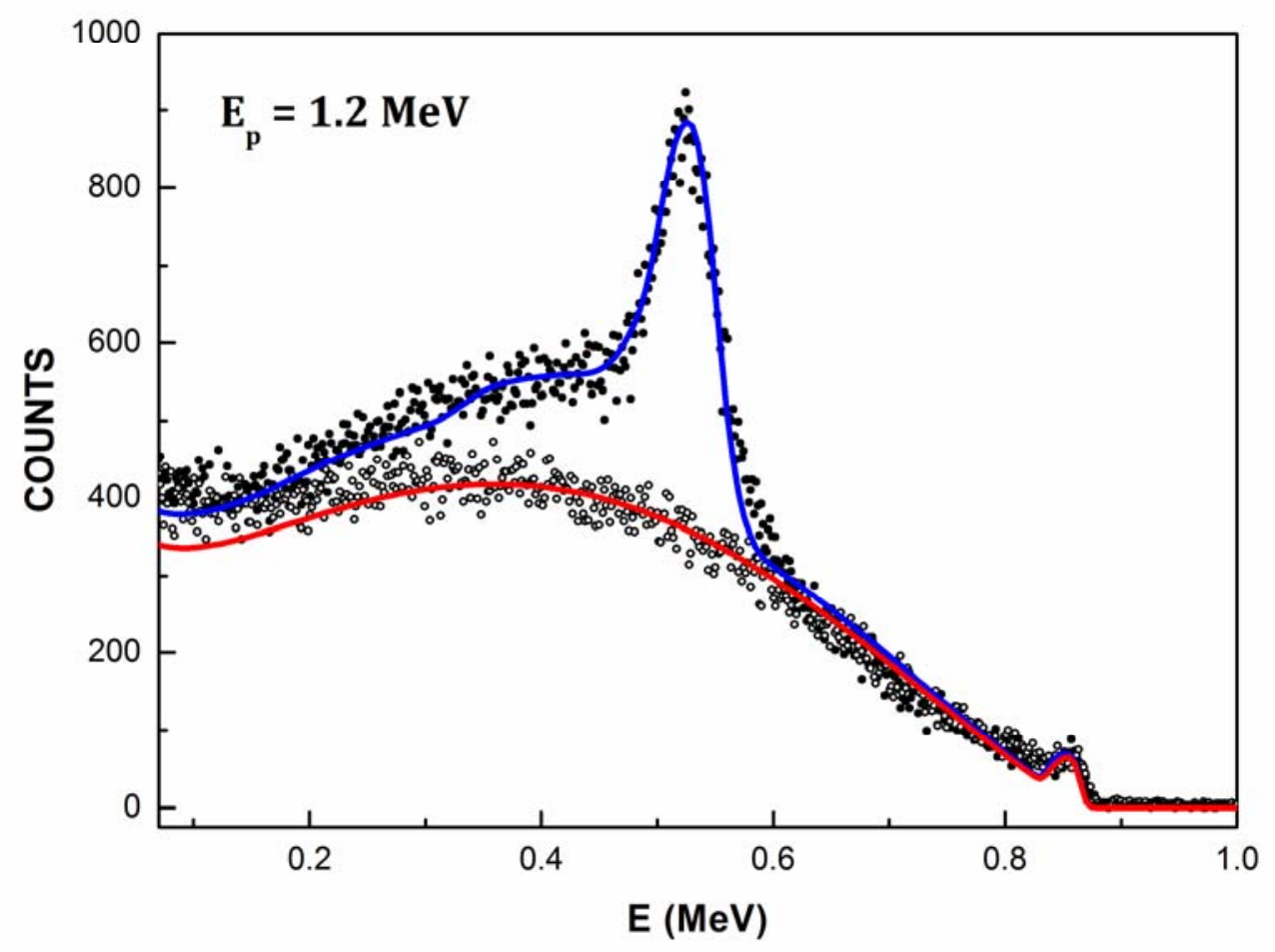

Figure 1. Experimental spectra for the implanted spots - black dots, for the virgin spots open dots, for the implanted spots - blue lines, for the virgin spots - red lines, in the case of $<100>$ diamond crystal and proton energies: (a) $1.2 \mathrm{MeV}$, (b) $1.5 \mathrm{MeV}$, (c) $1.75 \mathrm{MeV}$ and (d) $1.9 \mathrm{MeV}$. 


\section{Figure 1b}

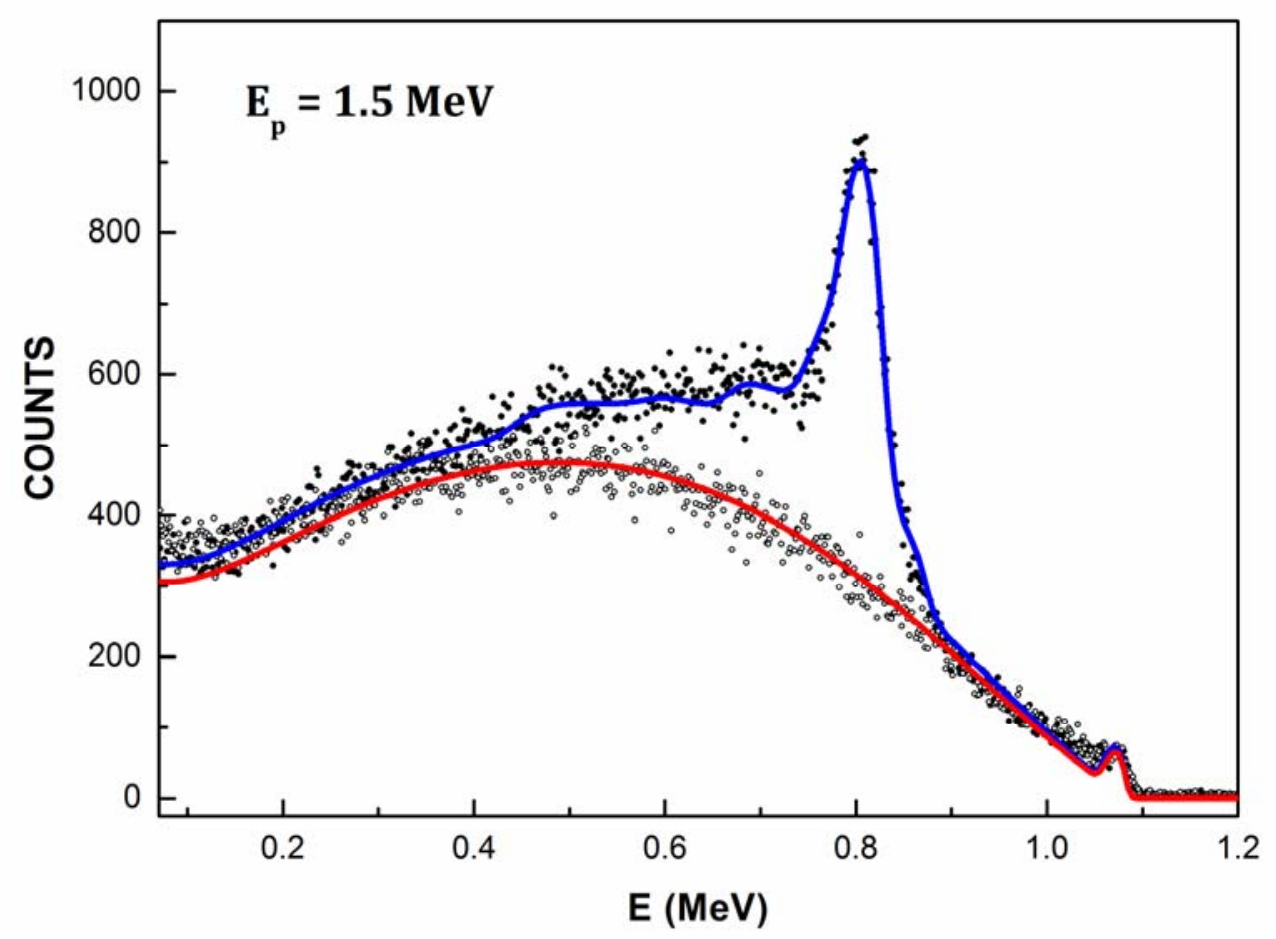

Figure 1. Experimental spectra for the implanted spots - black dots, for the virgin spots open dots, for the implanted spots - blue lines, for the virgin spots - red lines, in the case of $<100>$ diamond crystal and proton energies: (a) $1.2 \mathrm{MeV}$, (b) $1.5 \mathrm{MeV}$, (c) $1.75 \mathrm{MeV}$ and (d) $1.9 \mathrm{MeV}$. 
Figure 1c

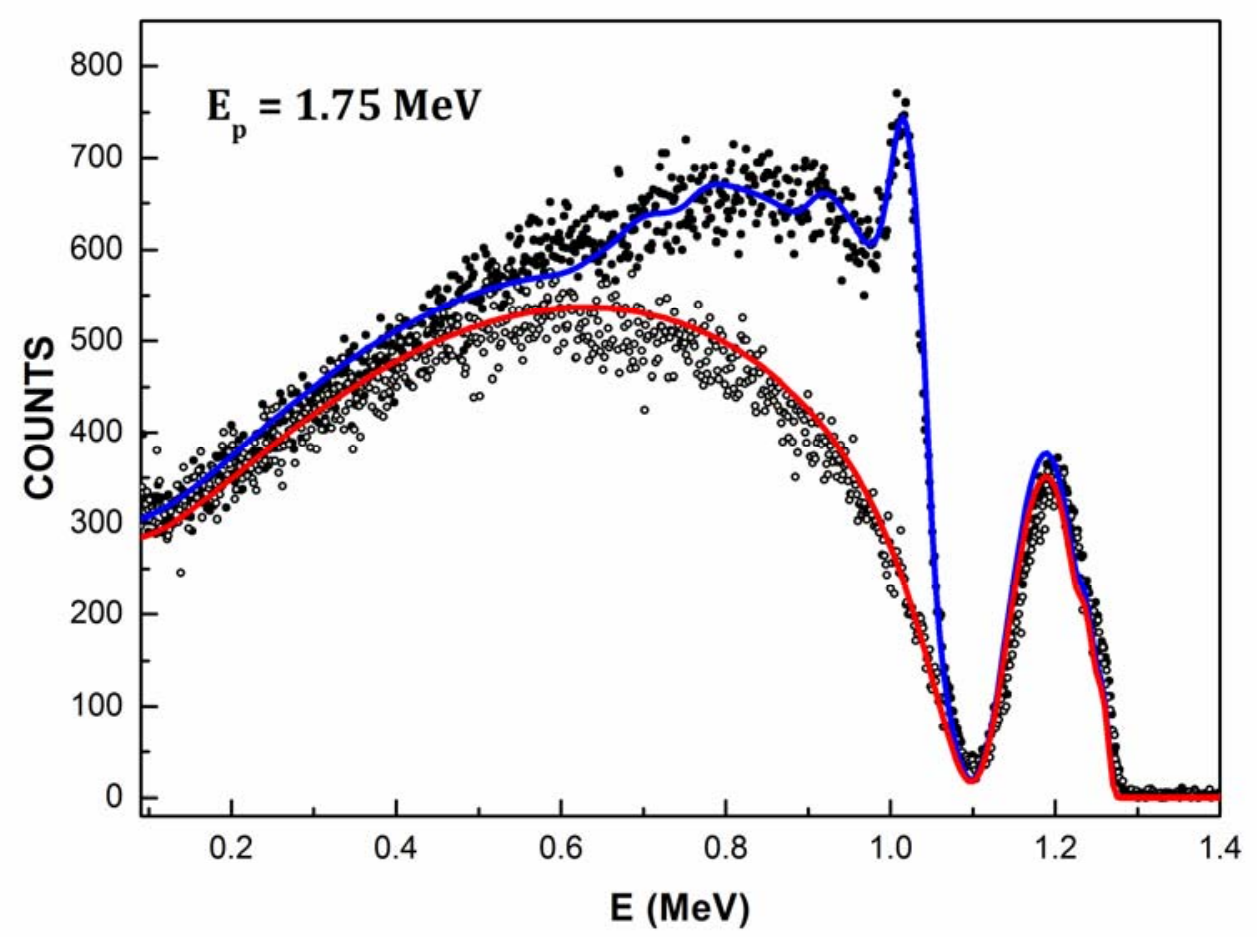

Figure 1. Experimental spectra for the implanted spots - black dots, for the virgin spots open dots, for the implanted spots - blue lines, for the virgin spots - red lines, in the case of <100> diamond crystal and proton energies: (a) $1.2 \mathrm{MeV}$, (b) $1.5 \mathrm{MeV}$, (c) $1.75 \mathrm{MeV}$ and (d) $1.9 \mathrm{MeV}$. 
Figure 1d

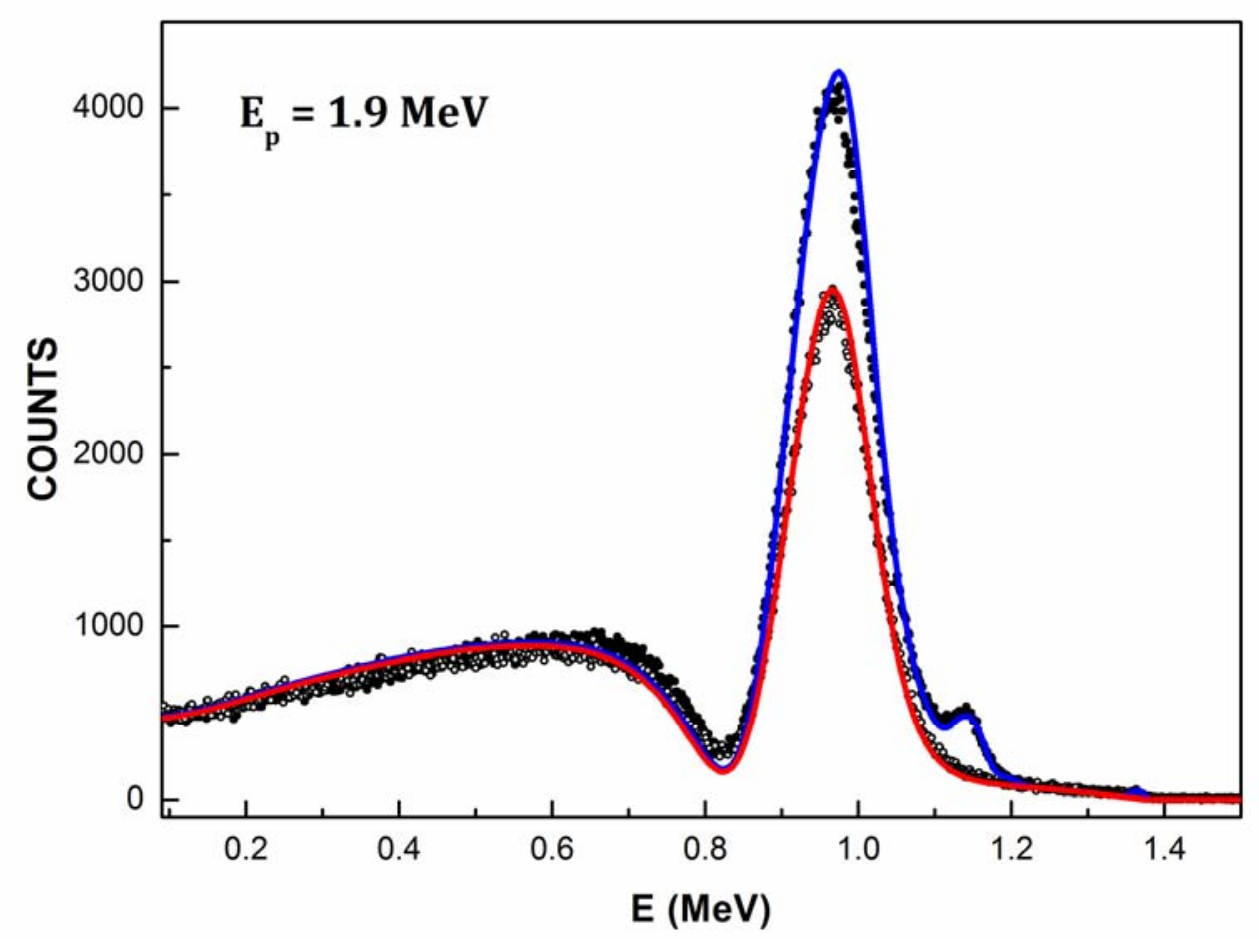

Figure 1. Experimental spectra for the implanted spots - black dots, for the virgin spots open dots, for the implanted spots - blue lines, for the virgin spots - red lines, in the case of <100> diamond crystal and proton energies: (a) $1.2 \mathrm{MeV}$, (b) $1.5 \mathrm{MeV}$, (c) $1.75 \mathrm{MeV}$ and (d) $1.9 \mathrm{MeV}$. 
Figure 2a

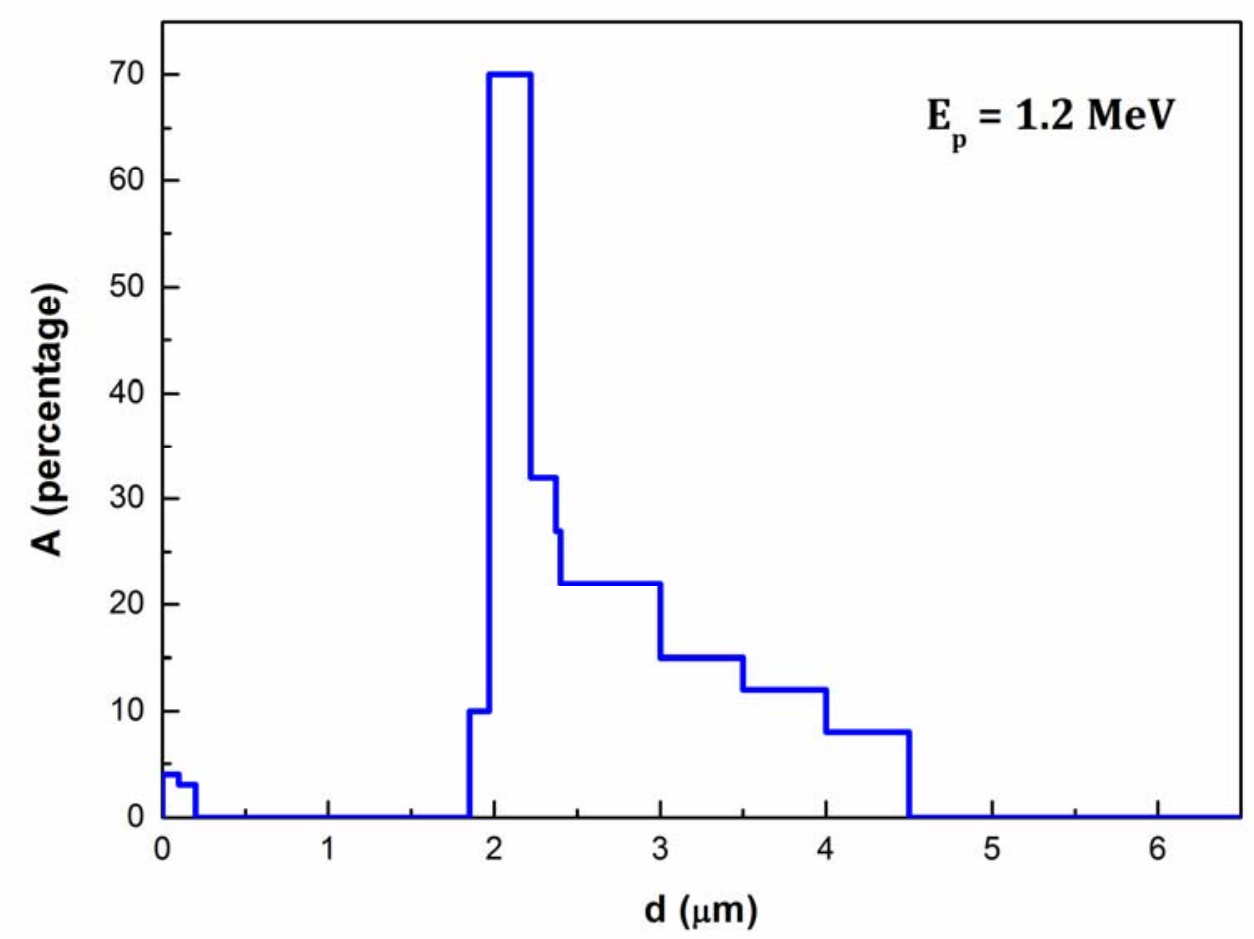

Figure 2. Amorphization depth profiles - blue lines, in the case of $<100>$ diamond crystal and proton energies: (a) $1.2 \mathrm{MeV}$, (b) $1.5 \mathrm{MeV}$, (c) $1.75 \mathrm{MeV}$ and (d) $1.9 \mathrm{MeV}$, corresponding to the simulation spectra for the implanted spots. 
Figure $2 b$

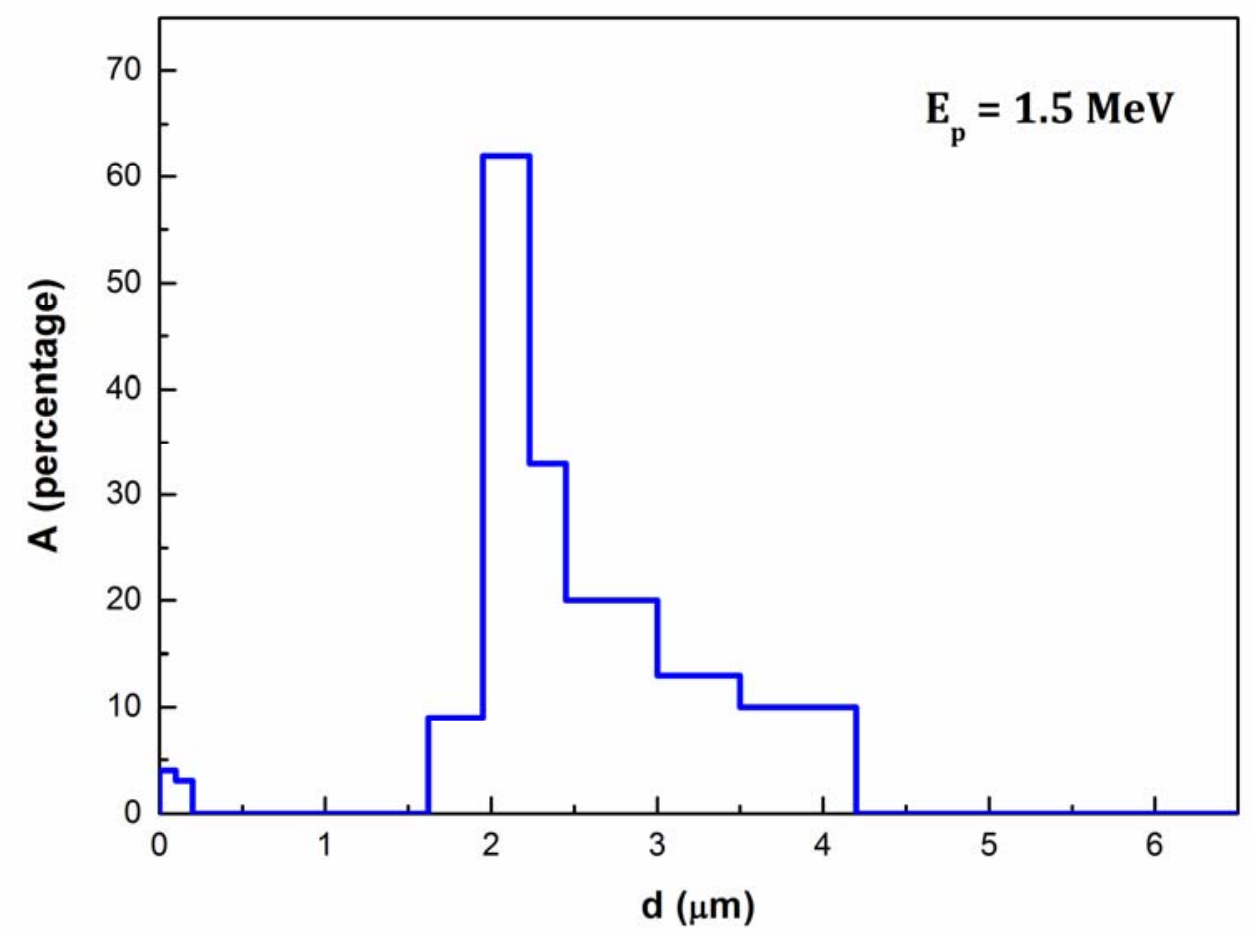

Figure 2. Amorphization depth profiles - blue lines, in the case of $<100>$ diamond crystal and proton energies: (a) $1.2 \mathrm{MeV}$, (b) $1.5 \mathrm{MeV}$, (c) $1.75 \mathrm{MeV}$ and (d) $1.9 \mathrm{MeV}$, corresponding to the simulation spectra for the implanted spots. 
Figure 2c

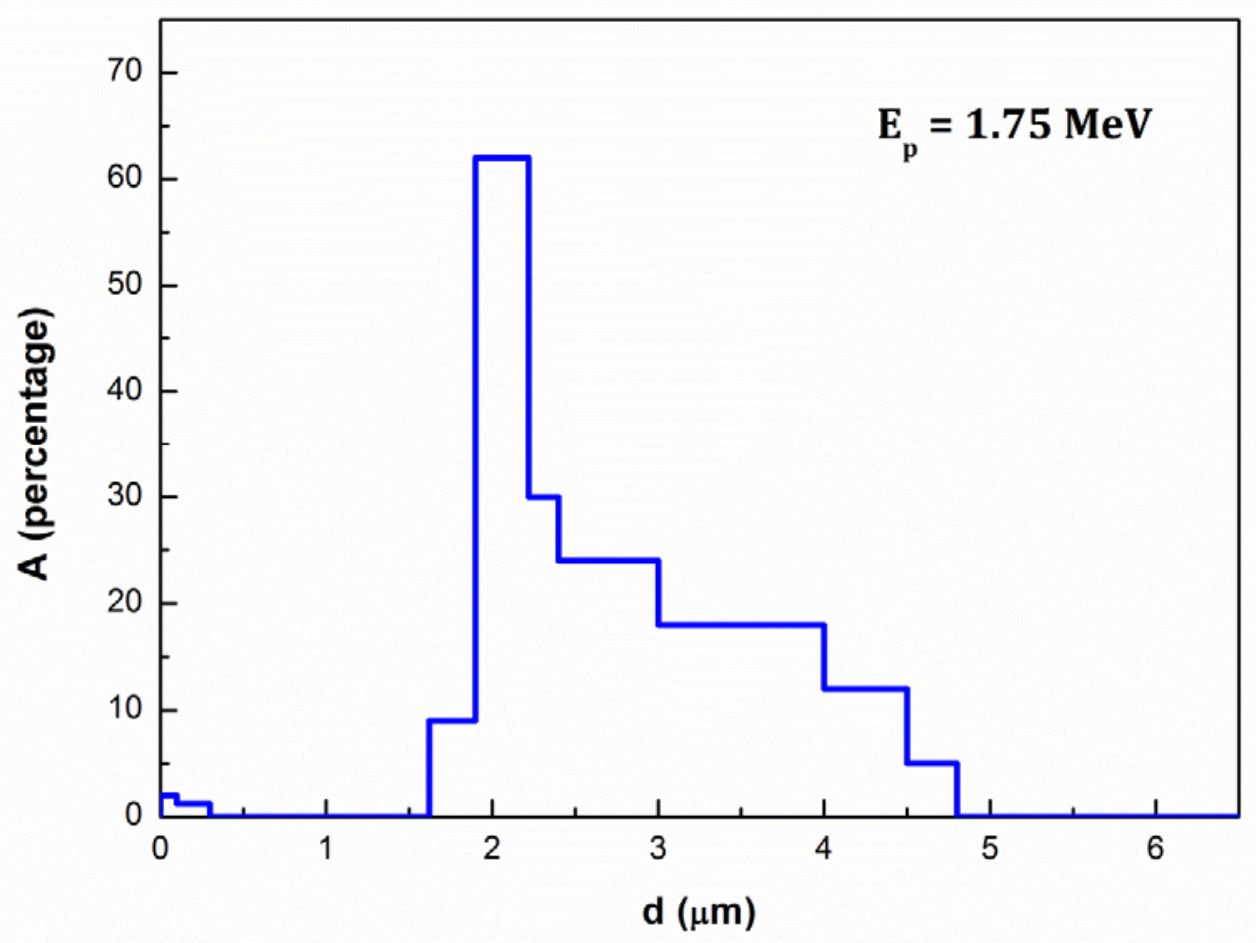

Figure 2. Amorphization depth profiles - blue lines, in the case of $<100>$ diamond crystal and proton energies: (a) $1.2 \mathrm{MeV}$, (b) $1.5 \mathrm{MeV}$, (c) $1.75 \mathrm{MeV}$ and (d) $1.9 \mathrm{MeV}$, corresponding to the simulation spectra for the implanted spots. 
Figure 2d

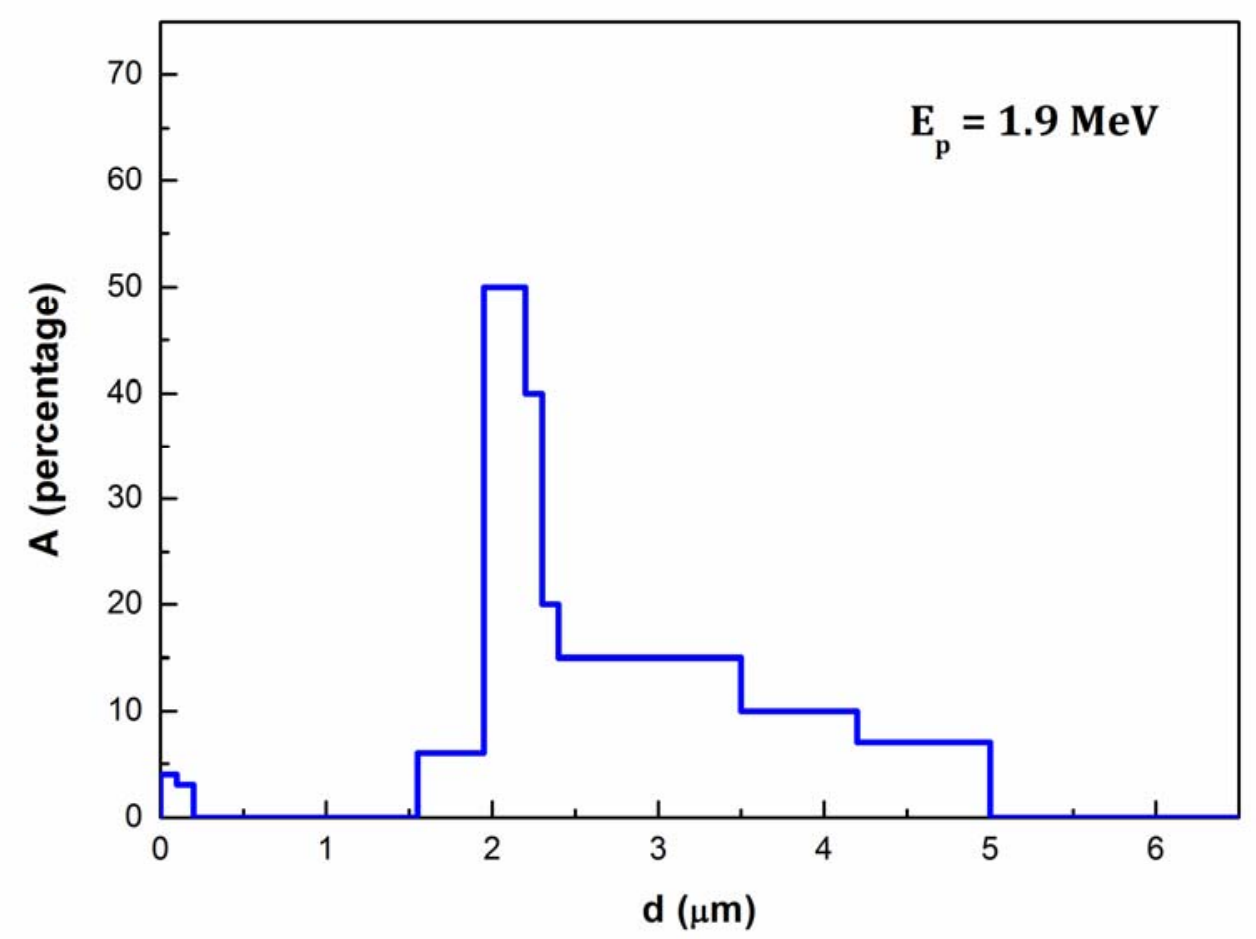

Figure 2. Amorphization depth profiles - blue lines, in the case of $<100>$ diamond crystal and proton energies: (a) $1.2 \mathrm{MeV}$, (b) $1.5 \mathrm{MeV}$, (c) $1.75 \mathrm{MeV}$ and (d) $1.9 \mathrm{MeV}$, corresponding to the simulation spectra for the implanted spots. 
Figure 3

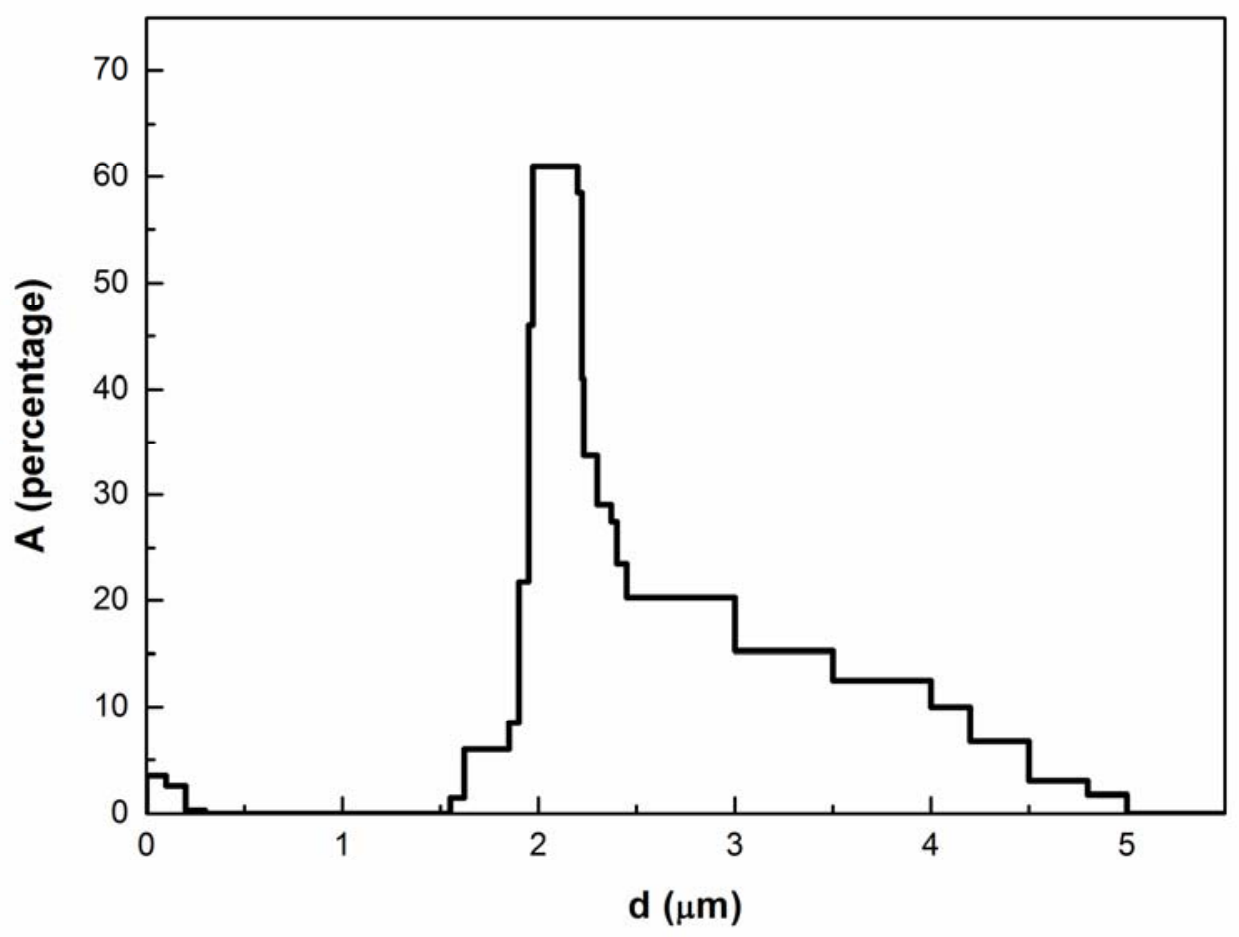

Figure 3. Averaged amorphization depth profile - black line. 